\title{
Novel pharmacological approaches to combat obesity and insulin resistance: targeting skeletal muscle with 'exercise mimetics'
}

\author{
A. L. Carey • B. A. Kingwell
}

Received: 9 February 2009 /Accepted: 5 May 2009/Published online: 23 June 2009

(C) Springer-Verlag 2009

\begin{abstract}
Chronic diseases arising from obesity will continue to escalate over coming decades. Current approaches to combating obesity include lifestyle measures, surgical interventions and drugs that target weight reduction or the metabolic consequences of obesity. Lifestyle measures including physical activity are usually the primary strategy, but these are of limited longterm efficacy because of failure to maintain behavioural change. An alternative approach used to elicit the benefits of exercise training and overcome the problems of long-term compliance is to develop drugs that mimic aspects of the trained state. Elucidation of metabolic pathways responsive to exercise in various tissues, particularly skeletal muscle, was an important antecedent to the promising concept of drugs that may mimic specific aspects of the exercise response. From an obesity perspective, an important aim is to develop an agent that reduces body fat and improves metabolic homeostasis. This review focuses on promising metabolic signalling pathways in skeletal muscle that may yield 'exercise mimetic' targets.
\end{abstract}

Keywords AMPK $\cdot$ Metabolic syndrome $\cdot$ PGC- $1 \alpha$.

Physical activity $\cdot$ PPAR $\cdot$ Review $\cdot$ SIRT1 $\cdot$ Skeletal muscle $\cdot$ Training · Type 2 diabetes

\footnotetext{
A. L. Carey $(\varangle) \cdot$ B. A. Kingwell

Metabolic and Vascular Physiology Laboratory,

Baker IDI Heart and Diabetes Institute,

PO Box 6492, St Kilda Road Central,

Melbourne VIC 8008, Australia

e-mail: andrew.carey@bakeridi.edu.au
}

\author{
Abbreviations \\ AICAR 5-Aminoimidazole-4-carboxamide-1- $\beta$-D- \\ ribofuranoside \\ AMPK 5'AMP-activated protein kinase \\ MAPK Mitogen-activated protein kinase \\ PEPCK Phosphoenolpyruvate carboxykinase \\ PGC- $1 \alpha$ Peroxisome proliferator-activated receptor- $\gamma$ \\ coactivator- $1 \alpha$ \\ PPAR Peroxisome proliferator-activated receptor \\ SIRT Silent information regulator of transcription
}

\section{Introduction}

Chronic diseases arising from obesity will continue to escalate over coming decades. The cost will be measured not only on a personal and population health level, but also on a global economic scale. Currently, $\sim 8 \%$ of the global population are obese and $\sim 25 \%$ are overweight, figures that are expected to rise an additional $\sim 50 \%$ within 10 years. Further, in developed regions (e.g. North America, Western Europe, Australia) obesity prevalence already approaches $25 \%$ [1]. In the UK, current estimates suggest that the population and gross domestic product will rise by $5 \%$ and 2.5 -fold, respectively, by 2050, whereas costs associated with obesity-related disease will rise by $>15$-fold [1]. Regular exercise is well recognised for its health promoting effects and a better understanding of the molecular mechanisms causing these beneficial effects is an excellent basis for identifying new therapeutic targets to combat metabolic and vascular disease.

The concept that regular exercise training is essential for maintaining optimal health and preventing metabolic 
disease is not new. Ancient Greek physicians, most notably through the teachings of Hippocrates, proposed that a lack of physical exertion was one of the primary causes of obesity [2]. More recently, in the early 20th Century, one of the forefathers of diabetes research, Elliot P. Joslin, similarly proposed that along with controlled diet and insulin therapy, regular exercise was a cornerstone of diabetes management [3]. Over the past 30 to 40 years, definitive studies using approaches ranging from epidemiological to interventional and molecular have proven, as reviewed [4], that regular exercise is effective in preventing and delaying metabolic disease and its complications.

The term 'exercise training' is somewhat generic and requires clarification. Different forms of exercise elicit specific biochemical responses, but can generally be categorised as aerobic/endurance or resistance/strength training. Both of these, when performed moderately and regularly, are beneficial for multiple disease states. While the ability to pharmacologically enhance muscular strength is a worthwhile pursuit for many disease states, including metabolic diseases such as type 2 diabetes, the recent quest for 'exercise mimetic' drugs for the treatment of obesity-related metabolic disease has primarily focussed on endurance exercise capacity concomitant with enhancement of energy expenditure and metabolic homeostasis. Since pharmaceutical targets primarily revolve around enhancement of endurance characteristics, for the purposes of this review we use the terms 'exercise' and 'exercise-mimetics' only with respect to endurance-based exercise training.

Currently available therapies for the treatment of obesity and related metabolic disorders

A number of therapeutic options are currently available for the treatment of obesity and metabolic dysregulation. While these provide variable degrees of benefit, currently none are free of disadvantages (see text box). Lifestyle modifications including diet and exercise are effective; however, sustained benefits are difficult to achieve due to problems in maintaining behavioural change. Pharmacological and/or surgical approaches are available to combat obesity for patients who fail to respond to lifestyle measures. There has been a recent rise in bariatric procedures to reduce the capacity for food intake and/or divert sections of the gastrointestinal tract, as reviewed comprehensively $[5,6]$. These procedures are highly effective at reducing body mass, improving insulin sensitivity and other aspects of the metabolic syndrome [5, 6]. Improvements in metabolic homeostasis also occur independently of body mass reduction [7], and given that conventional lifestyle measures are associated with poor compliance, surgical interventions are the most effective treatment currently available for morbid obesity $[8,9]$. Furthermore, surgery is considered a cost-effective measure in relation to the long-term healthcare costs of type 2 diabetes and its complications $[8,10]$. However, on a population scale, where in certain western countries overweight and obesity prevalence currently stand at $60 \%$ and $25 \%$, respectively, surgery alone is not a viable approach [11].

Current pharmacological approaches to obesity are limited. Orlistat, a gastrointestinal lipase inhibitor, sibutramine, a monamine-reuptake inhibitor and rimonabant, the first of the endocannabinoid receptor antagonists result in weight losses of up to $5 \mathrm{~kg}$ [11]. However, all are associated with unwanted side effects and none have resulted in sustained effective weight loss. The central melanocortin pathway, involved in regulating food intake, has also shown potential as an anti-obesity target, but substantial clinical development is still required [11]. While insulin secretagogues show promise for the management of glycaemia in the short term and better tolerated compounds have been recently identified, this class of drug neither significantly stems the deterioration in peripheral insulin sensitivity nor prevents loss of beta cell function [12]. Insulin sensitisers such as thiazolidinediones and metformin, which are used to treat the metabolic complications of obesity, are useful adjunct treatments for type 2 diabetes, but are neither free of side effects nor considered as effective as lifestyle modifications or surgical techniques for controlling metabolic disease [9, 13-17]. The extensive side effects (outlined in the text box) resulting from currently available pharmacotherapies are an important factor with respect to chronic treatment and therefore their ability to sustain long-term benefit. Further, most treatments available today are not without some level of interdependence on others for prolonged success, thereby accumulating additional unwanted side effects. In light of the lack of successful weight-loss treatments and the public health implications of the obesity pandemic, the development of safe and effective drugs is a major priority.

The elucidation of metabolic pathways responsive to exercise in various tissues, most particularly skeletal muscle, was an important antecedent to the promising concept of exercise mimetic drugs. From an obesity perspective, a pivotal aim would be development of an agent that improves energy expenditure while concomitantly reducing body fat and improving metabolic homeostasis.

Why target exercise-linked signalling?

An active lifestyle has well-documented health benefits. Aside from orthopaedic complications that are directly 
attributable to both volume of physical activity and body mass, and a small proportion of individuals whose insulin sensitivity or aerobic capacity do not improve as a result of aerobic training, exercise has virtually no disadvantages [18]. While the best-known effects of regular exercise are body weight control or, more simply, expenditure of energy, it is not necessary for overweight individuals to decrease body or adipose tissue mass to improve metabolic homeostasis [7, 19]. Accordingly, regular exercise results in adaptations including: (1) increased skeletal muscle oxidative capacity; (2) alterations in intracellular proteins and lipids involved in cellular signalling; and (3) cardiovascular adaptations that result in improved muscle and whole body insulin sensitivity, fuel partitioning and cardiovascular function. All of the above lead to prevention of metabolic disease $[20,21]$.

Evolutionarily humans have been programmed to minimise energy expenditure and in the developed world this trait has resulted in a largely sedentary population. In combination with an energy-rich environment, the consequence has been an escalation in obesity and chronic disease. Despite government and community initiatives to increase physical activity, participation and compliance rates are low, particularly once obesity and chronic disease are established [22, 23]. The poor adherence rate stems from more than just an evolutionary drive to remain sedentary; there are additional modern-day concerns such as socioeconomic status, ethnicity, age, sex, physical frailty and other sociological issues. These issues highlight the need for alternative approaches to reduce obesity and its consequences, while maintaining a focus on the molecular targets linked to the beneficial effects of exercise.

\section{Potential exercise-related targets}

Acute exercise training modulates the actions, in skeletal muscle, of enzymes, transcription factors, transporters and chaperones thought to control the long-term adaptive responses to chronic training and include: 5'AMP-activated protein kinase (AMPK) (recently reviewed in [24]), p38-mitogen-activated protein kinase (MAPK) [25], heat shock proteins [26, 27], peroxisome proliferator-activated receptor (PPAR)- $\gamma$ coactivator- $1 \alpha$ $(\mathrm{PGC}-1 \alpha)[28,29]$, nuclear factor $\mathrm{KB}$ [30], GLUT4 [31], fatty acid translocase/CD36 [32], calcium/calmodulindependent protein kinase (CAMK) II [33, 34], certain protein kinase $\mathrm{C}$ isoforms $[35,36]$, c-Jun $\mathrm{N}$-terminal kinase [25] and myokines (e.g. IL-6) [37] (Fig. 1). In general, these adaptive processes have been associated with health benefits. Importantly, for some of these factors, e.g. AMPK [38-41], heat shock protein 72 [42],
GLUT4 [31], PGC-1 $\alpha$ [43], protein kinase C- $\lambda$ [35, 44] and calcium signalling [45], transgenic, knockout and/or pharmacological activation studies in experimental animals have implied that manipulation of the relevant pathways in a pattern mimicking exercise may result in beneficial metabolic responses. In some examples, interestingly, the opposite is true: mouse lines in which signalling through c-Jun N-terminal kinase [46] and nuclear factor $\mathrm{kB}$ [47] modified clearly indicated that a reduction in activity improved insulin resistance, in contrast to the majority of data available, which, as reviewed [48], suggest that acute exercise activates these pathways. It is likely that such discordance arises from the nature of the exercise stimulus, which transiently activates these factors followed by rapid post-exercise inactivation. This contrasts with mouse transgenic models and chronic inflammatory states, which result in sustained effects and thus do not accurately model the episodic nature of exercise. This highlights an important consideration with respect to mimicking the effects of exercise, namely that, in some instances, the level of activity of factors during rest periods in the trained state should be mimicked, rather than the activity of these same factors during an acute exercise bout. This highlights the complexity of metabolic homeostasis and the need for comprehensive characterisation of exercise signalling pathways and their manipulation.

Of the factors listed above, those that are activated by exercise, such as AMPK, heat shock proteins, PGC- $1 \alpha$, calcium-associated signalling and certain MAPKs, are the most logical targets for pharmacological modulation to mimic the biochemical response/s to exercise. A second category includes those that are known to increase exercise capacity when overexpressed in muscle, or are activated pharmacologically or knocked-out, but are not necessarily known to be highly modulated by acute exercise training itself. The most prominent of this group are phosphoenolpyruvate carboxykinase (PEPCK)-C [49], silent information regulator of transcription (SIRT)1 [50], PPAR $\delta$ [51] and steroid receptor co-factor 3 [52]. Of particular additional interest is the fact that the studies cited for this group all observed a reduction of high-fat diet-induced obesity during the given interventions. The role of PEPCK-C in skeletal muscle function is rather obscure [49], while its role in regulation of hepatic gluconeogenesis suggests that pharmacological targeting as an exercise mimetic may be fraught with difficulty. However, activation of SIRT1 and PPAR $\delta$, as well as knockout of the gene encoding steroid receptor co-factor 3 were shown to target a broad spectrum of metabolic regulatory enzymes and genes, including certain 'exercise' factors listed above. From a pharmacological perspective, such effects could be considered non-specific, but if 


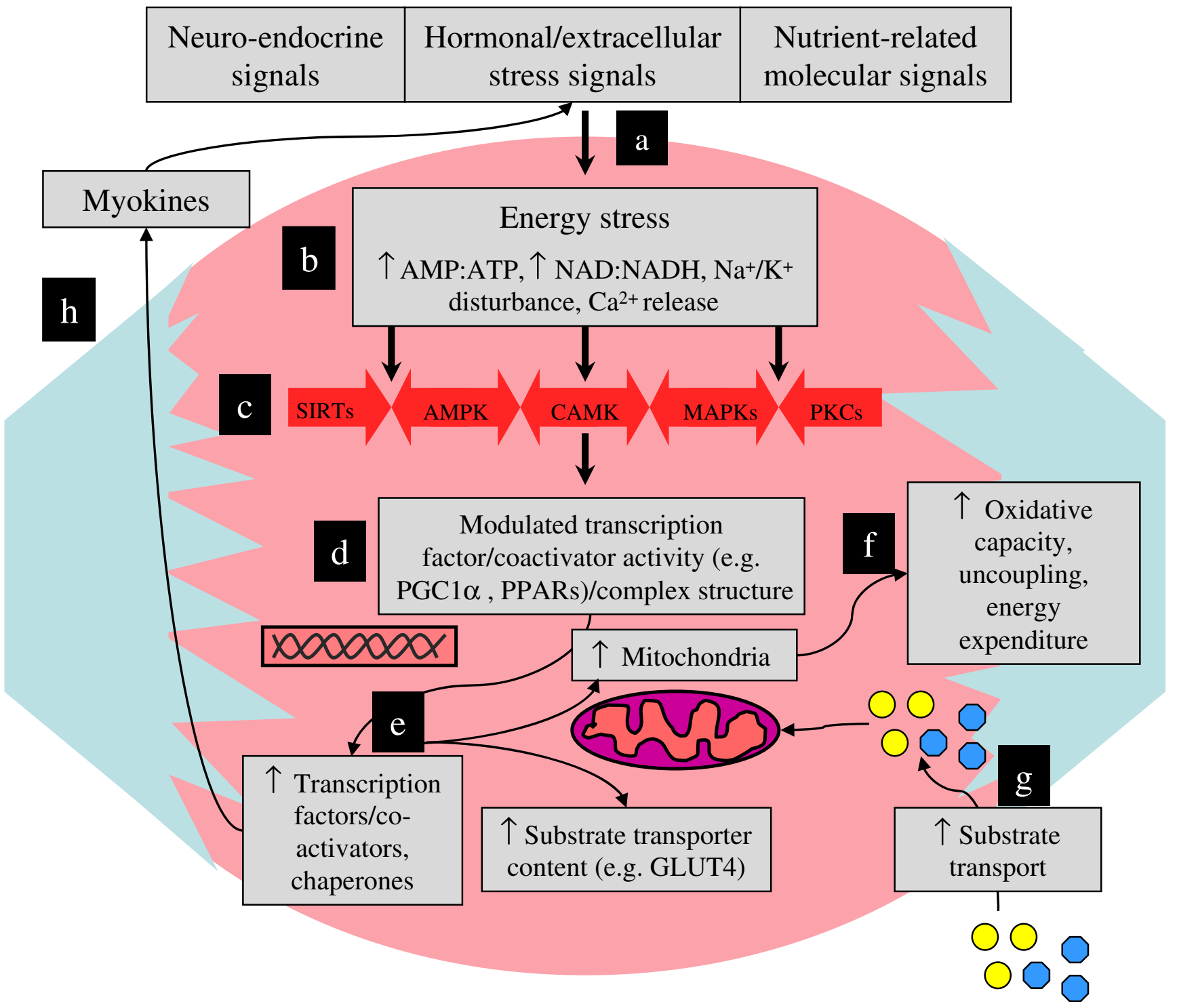

Fig. 1 Schematic overview of signals involved in mediating the adaptive responses of skeletal muscle to chronic endurance exercise training as well as putative targets of proposed therapeutic interventions that might mimic these responses. Numerous signals arise from the nervous system and blood stream (a), as well as via intracellularderived disturbances in homeostasis (b). The signals result in activation of several enzymes that are sensitive to this stimulus and, at certain levels, can interact with each other (c). These enzymes interact with transcription factors and coactivators (d), and via post-

targeted appropriately may mimic multiple aspects of the exercise response.

\section{Current candidate targets}

A number of approaches have been used recently to modulate the activity of certain molecules linked to exercise-related signalling processes. Promising results have been shown with respect to their ability to translational modifications result in modulation of nuclear transcriptional complexes, which increase the content of various factors that upregulate processes involved in regulation of substrate transport, storage and oxidation (e, f, $\mathbf{g})$. Contraction-linked signalling can also increase the production of muscle-derived secretory proteins (e.g. myokines), which may further contribute to the autocrine, paracrine or endocrine regulation of exercise metabolism (h), thereby providing additional pharmacological targets. CAMK, calcium/calmodulin-dependent protein kinase

improve both exercise capacity and metabolic health. These target molecules are discussed below.

PPAR $\delta$ PPAR $\delta$ is present in rodent and human skeletal muscle, and clear roles in muscle fatty acid and glucose oxidation, as well as oxidative gene expression have been demonstrated [30, 53]. Ppard overexpression prevents obesity [54], while skeletal muscle-specific overexpression causes a transition towards increased type 1 
(oxidative) muscle fibres and enhanced endurance exercise capacity [51] in mice. These data prompted Narkar et al. [55] to determine whether treating mice with a specific PPAR $\delta$ agonist (GW1516) for 4 weeks could induce similar effects. The primary finding was that while the PPAR $\delta$ agonist increased the expression of multiple skeletal muscle genes putatively responsible for increasing endurance exercise capacity, it neither increased the proportion of type 1 muscle fibres, nor increased exercise capacity. The authors, however, did not report on whether GW1516 prevented obesity [55]. A second study examining the combined effects of exercise training and GW1516 treatment elicited an extraordinary improvement in endurance exercise capacity and concomitant transition to oxidative fibres when compared with exercise training alone [55]. These results suggest that exercise activates another signalling cascade which interacts with PPAR $\delta$ stimulation to elicit a training response. Given the poor adherence to exercise training programmes in obese patients, this approach is thus limited, but could accelerate the adaptive response to training if used clinically in conjunction with an exercise programme. In addition, more potent PPAR $\delta$ activators may hold promise with respect to reproducing the anti-obesity actions of the transgenic model [54].

Clinically, single nucleotide polymorphisms in the PPARD gene have been identified that modify muscle glucose transport [56] and are linked to the level of improvement in aerobic capacity and insulin sensitivity seen after a training programme [57]. GW1516 treatment in monkeys improves blood lipid and lipoprotein profile [58], and has shown promise in small human trials with respect to improving metabolic homeostasis in obese patients, which was predominately attributed to improved skeletal muscle fatty acid oxidative capacity [59]. Further, training-induced PPAR $\delta$ protein content correlates well with improvements in clinical markers of type 2 diabetes [60], suggesting its levels in muscle are integral to the beneficial metabolic adaptations to training.

$A M P K$ AMPK is central to regulation of cellular metabolism and is activated when the energy charge of the cell is low, e.g. during energy deprivation and exercise [61]. Much research suggests some role of AMPK in regulation of exercise metabolism, including modulation of fibre type shift, gene expression and adaptation to training in either whole body or musclespecific Ampk 22 (also known as Prkaa2) knockout mice [24]. However, it is only recently that AMPK activation has been studied in vivo with respect to exercise performance. A well-described activator of AMPK, 5-aminoimidazole-4-carboxamide-1- $\beta$-D-ribofuranoside (AICAR; also known as Z-riboside or AICA riboside), was studied in direct comparison with GW1516 with respect to endurance capacity in mice [55]. After 4 weeks of AICAR therapy, exercise capacity was significantly enhanced in the absence of exercise training. This study also provided evidence to suggest interaction between the AMPK and PPAR $\delta$ pathways. This, along with our recent work demonstrating (1) that reconstituted HDL infusion in humans reduced plasma glucose, in part through activation of AMPK in skeletal muscle [62]; and (2) that blood lipid profile improved after GW1516 treatment in monkeys [58], further establishes an important clinical link between these exercise-regulated signalling pathways. Given that HDL is elevated in aerobically trained athletes, this may be one mechanism contributing to the enhanced glucose tolerance demonstrated by this group [63].

Narkar et al. [55] have validated the long-held belief that chronic AMPK activation can mimic some of the endurance-enhancing effects of exercise training and, importantly, that it is possible to pharmacologically enhance endurance and muscle oxidative capacity without the need for a simultaneous training programme. Importantly, while a previous study demonstrated that chronic AICAR treatment of obese rats did not reduce bodyweight and obesity, AICAR treatment was associated with increased skeletal muscle insulin-sensitivity and prevention of obesity-related complications, further validating its efficacy [64].

AMPK may be activated by endogenous nutrient signals. Indeed, since AMPK is traditionally described as a kinase activated by nutrient stress, this should come as no surprise. However, it is of interest that elevated concentrations of certain fatty acids can activate AMPK in vitro [65]. Furthermore, chronically elevated circulating fatty acids result in skeletal muscle mitochondrial biogenesis in rodents [66]. Whether manipulation of such signals, either endogenously or via exogenous means, is beneficial for energy expenditure and metabolic health is not known. This is of particular interest given that chronically elevated plasma fatty acid concentrations are known to be detrimental to metabolic homeostasis. Thus transient manipulation of certain fatty acids in the circulation may prove promising for improving tissue oxidative capacity and metabolic homeostasis.

Clinically, AICAR has proven efficacy when given intravenously to improve ischaemic reperfusion injury associated with coronary artery bypass graft surgery in Phase III clinical trials (acadesine) [67]. Further, an acute $(2 \mathrm{~h})$ intravenous infusion of AICAR in patients with type 2 diabetes resulted in favourable modulation of plasma glucose and NEFA concentrations, although neither all of the tissue-specific mechanisms, nor the AMPK-dependence of these effects could be elucidated [68]. Moreover, poor 
oral bioavailability limits application to acute intravenous indications. AMPK-activating agents with high oral bioavailability could have uses in treatment of impaired glucose tolerance, insulin resistance and types 1 and 2 diabetes. Indeed, metformin mediates at least part of its glucose-lowering actions through activation of AMPK [69]. A medicinal chemistry approach is therefore warranted, with a view to developing suitable compounds for preclinical trials of this potential application.

SIRT1 The naturally derived polyphenolic compound resveratrol, found most commonly in the skin of certain grape varieties, has for some time been known for its cardio-protective effects in mammals and life-extending properties in lower organisms [70]. Recently the deacetylase enzyme Sir2 and its mammalian homologue SIRT1 were identified as putative primary targets for resveratrol. In addition, resveratrol also activates AMPK in cell culture systems, although not via direct interaction [71]. Lagouge et al. [50] demonstrated that resveratrol treatment for 3 months enhanced exercise capacity, but also protected mice from obesity. The mechanisms involve deacetylation (activation) of PGC-1 $\alpha$, a key transcriptional coactivator integral to mitochondrial production [72] and a target of AMPK [73]. Considering that SIRT1 alters activity, either positively or negatively, via acetylation and that AMPK is not known to be regulated by acetylation, the mechanism by which resveratrol activates AMPK was until recently unclear. Lan et al. [74] have demonstrated that SIRT1 is able to activate the serine/threonine kinase LKB1, the best described upstream activating kinase of AMPK [75], therefore establishing strong molecular links between SIRT1, AMPK, PGC- $1 \alpha$ and PPAR $\delta$ (Fig. 2). Interestingly with respect to this signalling cascade, the novel polyphenolic compound SRT1720 was identified as a more potent SIRT1 activator than resveratrol and also shown to prevent obesity [76, 77] and increase endurance capacity [76] in a manner that appeared to be less dependent, but not entirely independent of AMPK. This implies that SRT1720 exhibits greater SIRT1 specificity via actions directed at deacetylation of important substrates such as PGC- $1 \alpha$, forkhead box O1 and $\mathrm{p} 53$, but that the indirect activation of AMPK may

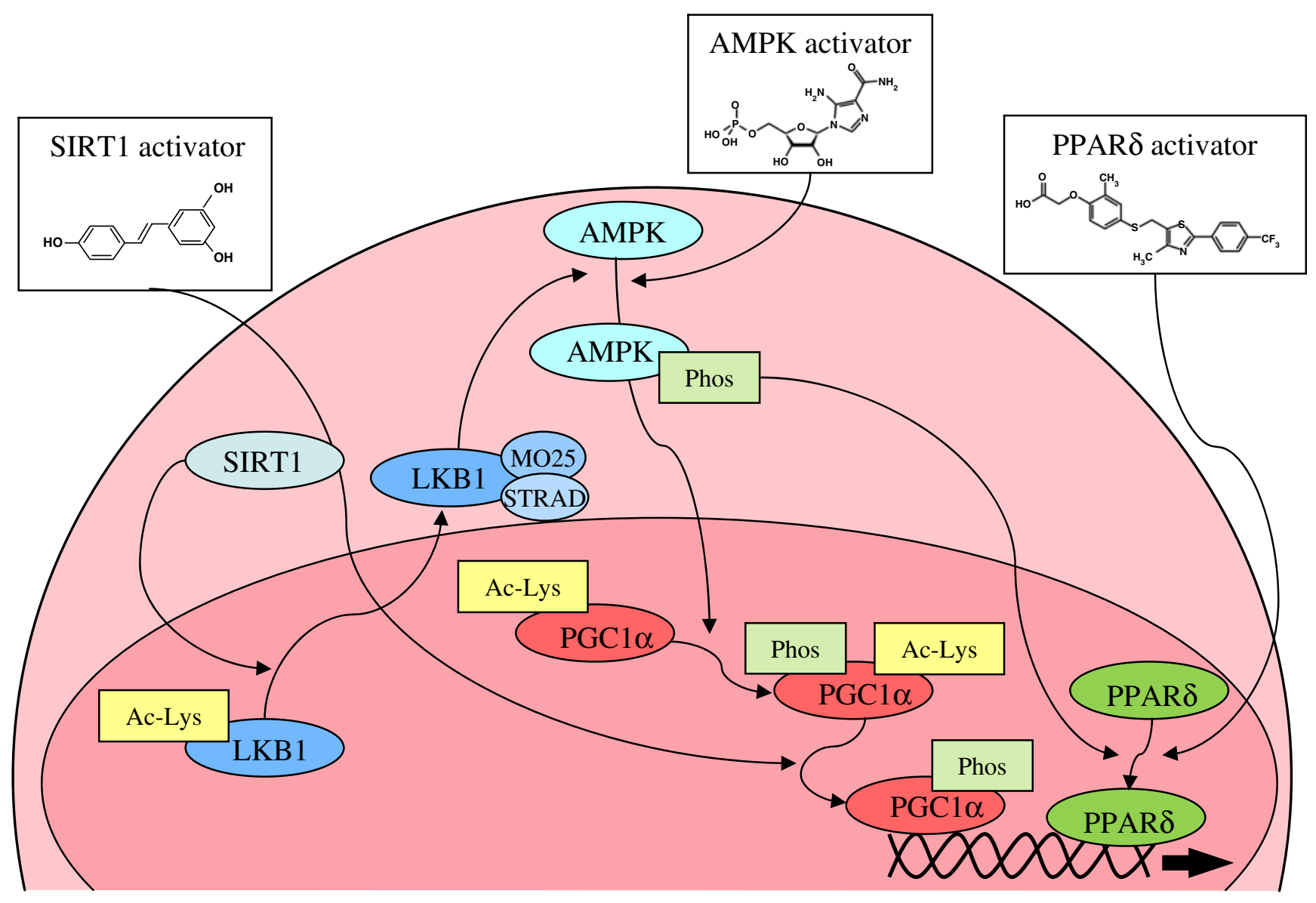

Fig. 2 The $\operatorname{link}(\mathrm{s})$ between signalling molecules reported to be involved in replication of an exercise-training phenotype via pharmacological activation. Mouse protein 25 (MO25) and Ste20-related adaptor protein (STRAD) are accessory proteins required for activation of LKB1. Ac-Lys, acetylation of lysine; Phos, phosphorylation 
be necessary for the full complement of beneficial effects [76]. SIRT1-activating compounds target multiple tissues [50, 76-78], raising the possibility that such agents have the potential to mimic multiple beneficial aspects of the training response rather than inducing a muscle-specific target. Little is known with respect to translation of SIRT1 function to human skeletal muscle, although restricted energy intake is known to increase muscle levels SIRT1 mRNA [79]. Novel SIRT1 activators have been developed $[76,77]$, with substantially greater potency than resveratrol; these can protect mice from diet-induced obesity and insulin insensitivity, and are among the most promising candidates for clinical application.

$P G C-1 \alpha$ The potential for activation of PGC- $1 \alpha$ as a molecular target for metabolic diseases has been highlighted above, since it is integral in co-ordinating downstream signals for at least some of the beneficial effects of the factors previously described. In addition, PGC- $1 \alpha$ is present in human skeletal muscle, is highly inducible by exercise training $[80,81]$ and polymorphisms of the gene encoding it have been identified, which independently [82] and in conjunction with a PPARD polymorphism [57, 83] correlate with insulin resistance, obesity and adaptability to training interventions. The concept that skeletal muscle PGC- $1 \alpha$ might be a potential pharmaceutical target based on knowledge of its modulation by exercise has been reviewed previously [84].

Key aspects, candidate targets On the basis of evidence available, it is apparent that with respect to pharmacological enhancement of skeletal muscle endurance exercise capacity, activation of AMPK provides a broader spectrum target than PPAR $\delta$ due to its upstream position in this signalling cascade. This applies even more so to SIRT1 in relation to AMPK, and it is clear that these signals are linearly linked. With this in mind, it could be argued that exercise mimetics should target regulatory molecules at the proximal end of signalling cascades so as to target multiple pathways eliciting the broad array of benefits associated with training. This is in contrast to conventional pharmacological approaches, which generally aim for specificity to reduce unwanted effects. The challenge for exercise mimetics will be replication of the subtleties of the exercise stimulus, including its periodic nature, in order to activate the desired pathways.

\section{Other candidates}

Numerous manipulations of metabolic regulatory genes in mice, a list far too broad for the scope of this review, have demonstrated protection from diet-induced obesity through increased energy expenditure, analogous to increasing skeletal muscle content of PEPCK-C and PPAR $\delta$. Conceivably the mechanism/s of action could involve exerciserelated signalling pathways, although most of these mouse models have not been assessed with regard to effects on endurance capacity.

Caffeine has been used for many years in sport as a performance enhancer, but most commonly as an acute stimulant. Evidence also persists for an acute ergogenic affect on muscle substrate metabolism; however this remains controversial [85]. Nevertheless caffeine activates diverse signalling pathways associated with adaptation to endurance exercise in cultured skeletal muscle. For example, caffeine increases calcium release from the sarcoplasmic reticulum [86] and also activates AMPK [87] and p38-MAPK [88] when administered in high, supra-physiological doses $(>3 \mathrm{mmol} / \mathrm{l})$. While it is freely consumed by athletes as well as the community at large in doses that can result in circulating concentrations $\sim 100$ fold lower than those seen in cell culture studies [85], there is no in vivo evidence regarding effects of chronic ingestion of caffeine on skeletal muscle function or endurance capacity.

Murase et al. [89] have shown in mice that 8 to 10 weeks ingestion of green tea extract, containing concentrated levels of various catechin molecules, increased endurance exercise performance when combined with exercise training. However, they did not report whether green tea extract alone enhanced endurance. Green tea catechins can activate AMPK in non-muscle cells [90, 91], suggesting a mechanism similar to other AMPK activators such as AICAR [55] and resveratrol [50]. However, Murase et al. [89] suggest that the actions of green tea extract in skeletal muscle are independent of PPAR $\alpha / \delta$ signalling, which contrasts with the purported mechanism of AICAR treatment [55]. Green tea extract/catechin ingestion could therefore be part of a potential exercise mimetic strategy; however, more work is required to determine the signalling mechanisms responsible, as well as whether green tea extract/catechin can act alone or only in concert with training.

Various antioxidant molecules and other essential nutrients are consumed as dietary supplements, for presumed health benefits, although most are unsubstantiated by clinical trial evidence. Given that many are structural analogues of resveratrol or catechins, many other molecules might be able to enhance exercise capacity when ingested chronically in large doses. Thus far, only ascorbic acid/ vitamin $\mathrm{C}$ has been tested in appropriately controlled studies and was found to impair adaptation to training in rodents and humans [92].

Myokines are a class of proteins produced and secreted by skeletal muscle. To date the only myokine yet to be clearly classified is interleukin-6, with limited evidence perhaps implicating interleukin-15 [37]. While there is no 
evidence that these or molecules with similar characteristics might improve exercise capacity, clear metabolic benefits have been identified that suggest molecules secreted by contracting skeletal muscle might be advantageous for skeletal muscle metabolic function and therefore harnessed for therapeutic use [93].

Finally, it is worth considering that drugs used illegally as performance enhancing agents in sport might have the potential to combat obesity and metabolic disease. When properly controlled, such agents could be applied to accelerate exercise adaptation and reduce the barriers to exercise in patients incapable of exercise. This and the development of new compounds that enhance skeletal muscle and other important metabolic organ function are likely to be productive avenues for future anti-obesity therapies.

\section{Mimicking endurance exercise: the down-side}

Mimicking activation of exercise signalling pathways could result in a chronic catabolic state. For example, activation of AMPK could inhibit protein synthesis [94] and stimulate autophagy [95]. Also, while augmenting oxidative capacity in mice, skeletal muscle overproduction of PGC- $1 \alpha$ resulted in severe muscle atrophy as the mice aged [96]. These effects would clearly be detrimental, especially for ageing patients. Potential therapeutics would need to strike an optimum balance between continuous compared with transient activation. Such negative actions suggest that exercise mimetics would be indicated as a short-term or adjunct treatment to evoke a negative energy balance and attainment of the desired target weight, which could then be maintained through lifestyle measures. Finally, the protective effects of SRT1720 treatment have been linked to modulation of brown adipose tissue function [76]. Since there is little evidence that exercise training impacts significantly on brown adipose tissue function, any treatment acting via this mechanism would not strictly be classified as an 'exercise mimetic'.

Mimicking exercise: an impossible task?

Intense, prolonged exercise bouts induce significant stress on various organ systems, most particularly, skeletal muscle. With a $>15$-fold increase in whole body oxygen consumption when transitioning from complete rest to intense dynamic exercise, it is no surprise that numerous biochemical signalling pathways are activated in multiple tissues. Importantly training has multiple health benefits that do not, at least directly or entirely, relate to this elevation in energy expenditure. First, many cardiovascular adaptations including blood pressure reduction and enhanced lipid profile are at least in part unrelated to muscle-specific adaptation [20, 97]. Additionally, other health benefits attributed to regular exercise include amelioration of: dementia and other neurological disorders, osteoarthritis, chronic obstructive pulmonary disease, osteoporosis, fall-related injuries, depression, certain cancers and forms of cardiovascular disease [2, 98]. Obviously, no single pharmaceutical agent could mimic this response. Furthermore, single agents, conditions or therapies that primarily target an increase in energy expenditure (e.g. hyperthyroidism, adrenergic receptor agonists or mitochondrial uncouplers) have consistently resulted in adverse side effects that render them unsuitable for therapeutic use. A polypill containing a number of agents aimed at selected targets is another possibility, but is likely to be associated with multiple unwanted effects and to be of questionable efficacy. It seems more likely that further understanding of the molecular pathways activated by exercise in various organ systems will yield tissue-specific targets enabling limited aspects of the exercise response to be mimicked. In this regard, the exercise-trained state may not necessarily be the goal; instead the focus should be simply on increasing whole body energy expenditure rather than a true exerciselike response. The term 'exercise mimetic' is thus quite generic and more descriptive terms should be applied to individual agents mimicking specific and limited aspects of the exercise response.

\section{The future}

Lifestyle modification, including reduced energy intake and elevated energy expenditure through physical activity, remains the most important approach to combat the current obesity epidemic on a population level. A focus on developing healthy lifestyle habits in childhood and maintaining these throughout life is particularly critical. There are, however, subpopulations, including the morbidly obese, who are unable to exercise and will require more aggressive interventions. An understanding of the signalling pathways activated by exercise in metabolically relevant tissue has already proven to be a productive avenue for identifying drug targets to combat obesity. While no single agent will ever mimic the broad range of exercise-related health benefits [22, 98-100], targeting of specific aspects of the exercise response is likely to yield health benefits. In light of this, use of the term 'exercise mimetic' is probably not appropriate; rather, a term that better reflects the specific aspect of exercise being targeted may achieve better acceptance. This area is still embryonic in terms of clinical development, and while targets have been identified, many challenges lie ahead with respect to development of formulations with appropriate pharmacological properties for chronic use with minimal side effects. 


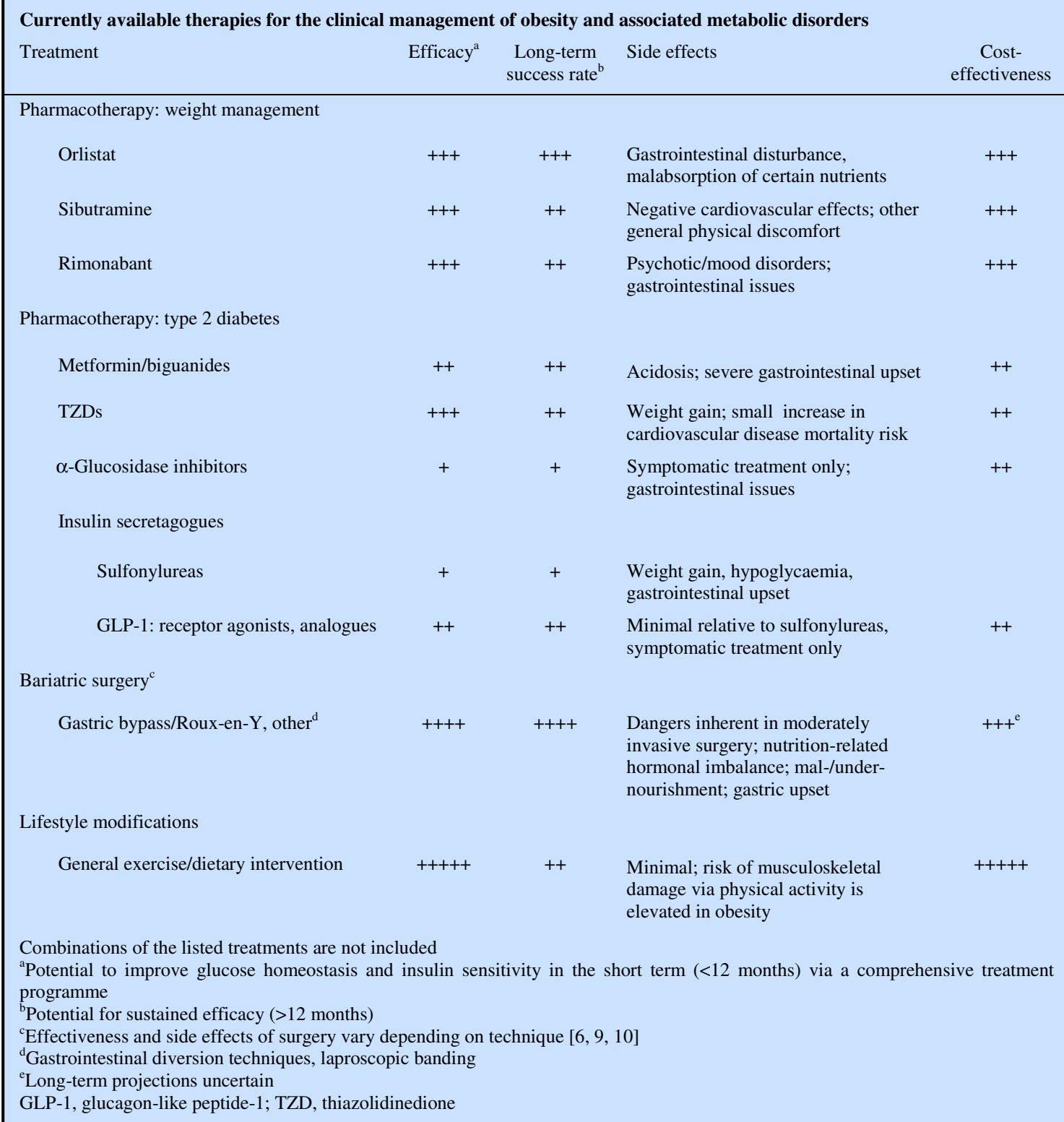

Duality of interest The authors declare that there is no duality of interest associated with this manuscript.

\section{References}

1. James WP (2008) The epidemiology of obesity: the size of the problem. J Intern Med 263:336-352

2. Booth FW, Lees SJ (2007) Fundamental questions about genes, inactivity, and chronic diseases. Physiol Genomics 28:146-157

3. Schneider SH, Ruderman NB (1986) Exercise and physical training in the treatment of diabetes mellitus. Compr Ther 12:49-56

4. Hamilton MT, Booth FW (2000) Skeletal muscle adaptation to exercise: a century of progress. J Appl Physiol 88:327-331
5. O’Brien PE, Brown WA, Dixon JB (2005) Obesity, weight loss and bariatric surgery. Med J Aust 183:310-314

6. Tice JA, Karliner L, Walsh J, Petersen AJ, Feldman MD (2008) Gastric banding or bypass? A systematic review comparing the two most popular bariatric procedures. Am J Med 121:885-893

7. Troy S, Soty M, Ribeiro L et al (2008) Intestinal gluconeogenesis is a key factor for early metabolic changes after gastric bypass but not after gastric lap-band in mice. Cell Metab 8:201-211

8. Clegg A, Colquitt J, Sidhu M, Royle P, Walker A (2003) Clinical and cost effectiveness of surgery for morbid obesity: a systematic review and economic evaluation. Int J Obes Relat Metab Disord 27:1167-1177

9. Dixon JB, O'Brien PE, Playfair J et al (2008) Adjustable gastric banding and conventional therapy for type 2 diabetes: a randomized controlled trial. JAMA 299:316-323 
10. Kushner RF, Noble CA (2006) Long-term outcome of bariatric surgery: an interim analysis. Mayo Clin Proc 81:S46-51

11. Padwal RS, Majumdar SR (2007) Drug treatments for obesity: orlistat, sibutramine, and rimonabant. Lancet 369:71-77

12. Raskin P (2008) Why insulin sensitizers but not secretagogues should be retained when initiating insulin in type 2 diabetes. Diabetes Metab Res Rev 24:3-13

13. Bolen S, Feldman L, Vassy J et al (2007) Systematic review: comparative effectiveness and safety of oral medications for type 2 diabetes mellitus. Ann Intern Med 147:386-399

14. Knowler WC, Barrett-Connor E, Fowler SE et al (2002) Reduction in the incidence of type 2 diabetes with lifestyle intervention or metformin. N Engl J Med 346:393-403

15. Lessard SJ, Rivas DA, Chen ZP et al (2007) Tissue-specific effects of rosiglitazone and exercise in the treatment of lipidinduced insulin resistance. Diabetes 56:1856-1864

16. Luft D, Schmulling RM, Eggstein M (1978) Lactic acidosis in biguanide-treated diabetics: a review of 330 cases. Diabetologia 14:75-87

17. Nissen SE, Wolski K (2007) Effect of rosiglitazone on the risk of myocardial infarction and death from cardiovascular causes. N Engl J Med 356:2457-2471

18. Teran-Garcia M, Rankinen T, Koza RA, Rao DC, Bouchard C (2005) Endurance training-induced changes in insulin sensitivity and gene expression. Am J Physiol Endocrinol Metab 288: E1168-E1178

19. Bruce CR, Kriketos AD, Cooney GJ, Hawley JA (2004) Disassociation of muscle triglyceride content and insulin sensitivity after exercise training in patients with Type 2 diabetes. Diabetologia 47:23-30

20. Jennings G, Nelson L, Nestel P et al (1986) The effects of changes in physical activity on major cardiovascular risk factors, hemodynamics, sympathetic function, and glucose utilization in man: a controlled study of four levels of activity. Circulation 73:30-40

21. Wojtaszewski JF, Richter EA (2006) Effects of acute exercise and training on insulin action and sensitivity: focus on molecular mechanisms in muscle. Essays Biochem 42:31-46

22. Goodyear LJ (2008) The exercise pill - too good to be true? N Engl J Med 359:1842-1844

23. Wing RR (1999) Physical activity in the treatment of the adulthood overweight and obesity: current evidence and research issues. Med Sci Sports Exerc 31:S547-S552

24. Richter EA, Ruderman NB (2009) AMPK and the biochemistry of exercise: implications for human health and disease. Biochem J 418:261-275

25. Goodyear LJ, Chang PY, Sherwood DJ, Dufresne SD, Moller DE (1996) Effects of exercise and insulin on mitogen-activated protein kinase signaling pathways in rat skeletal muscle. Am J Physiol 271:E403-E408

26. Hernando R, Manso R (1997) Muscle fibre stress in response to exercise: synthesis, accumulation and isoform transitions of 70 kDa heat-shock proteins. Eur J Biochem 243:460-467

27. Walsh RC, Koukoulas I, Garnham A, Moseley PL, Hargreaves M, Febbraio MA (2001) Exercise increases serum Hsp72 in humans. Cell Stress Chaperones 6:386-393

28. Baar K, Wende AR, Jones TE et al (2002) Adaptations of skeletal muscle to exercise: rapid increase in the transcriptional coactivator PGC-1. FASEB J 16:1879-1886

29. Goto M, Terada S, Kato M et al (2000) cDNA cloning and mRNA analysis of PGC-1 in epitrochlearis muscle in swimmingexercised rats. Biochem Biophys Res Commun 274:350-354

30. Kramer DK, Al-Khalili L, Guigas B, Leng Y, Garcia-Roves PM, Krook A (2007) Role of AMP kinase and PPARdelta in the regulation of lipid and glucose metabolism in human skeletal muscle. J Biol Chem 282:19313-19320
31. Rodnick KJ, Henriksen EJ, James DE, Holloszy JO (1992) Exercise training, glucose transporters, and glucose transport in rat skeletal muscles. Am J Physiol 262:C9-C14

32. Bonen A, Dyck DJ, Ibrahimi A, Abumrad NA (1999) Muscle contractile activity increases fatty acid metabolism and transport and FAT/CD36. Am J Physiol 276:E642-E649

33. Fluck M, Waxham MN, Hamilton MT, Booth FW (2000) Skeletal muscle $\mathrm{Ca}(2+)$-independent kinase activity increases during either hypertrophy or running. J Appl Physiol 88:352-358

34. Rose AJ, Frosig C, Kiens B, Wojtaszewski JF, Richter EA (2007) Effect of endurance exercise training on $\mathrm{Ca}^{2+}$ calmodulin-dependent protein kinase II expression and signalling in skeletal muscle of humans. J Physiol 583:785-795

35. Rockl KS, Witczak CA, Goodyear LJ (2008) Signaling mechanisms in skeletal muscle: acute responses and chronic adaptations to exercise. IUBMB Life 60:145-153

36. Rose AJ, Michell BJ, Kemp BE, Hargreaves M (2004) Effect of exercise on protein kinase $\mathrm{C}$ activity and localization in human skeletal muscle. J Physiol 561:861-870

37. Pedersen BK, Febbraio MA (2008) Muscle as an endocrine organ: focus on muscle-derived interleukin-6. Physiol Rev 88:1379-1406

38. Bergeron R, Previs SF, Cline GW et al (2001) Effect of 5-aminoimidazole-4-carboxamide-1-beta-D-ribofuranoside infusion on in vivo glucose and lipid metabolism in lean and obese Zucker rats. Diabetes 50:1076-1082

39. Hayashi T, Hirshman MF, Kurth EJ, Winder WW, Goodyear LJ (1998) Evidence for 5' AMP-activated protein kinase mediation of the effect of muscle contraction on glucose transport. Diabetes 47:1369-1373

40. Jorgensen SB, Treebak JT, Viollet B et al (2007) Role of AMPKalpha2 in basal, training-, and AICAR-induced GLUT4, hexokinase II, and mitochondrial protein expression in mouse muscle. Am J Physiol Endocrinol Metab 292:E331-E339

41. Jorgensen SB, Wojtaszewski JF, Viollet B et al (2005) Effects of alpha-AMPK knockout on exercise-induced gene activation in mouse skeletal muscle. FASEB J 19:1146-1148

42. Chung J, Nguyen AK, Henstridge DC et al (2008) HSP72 protects against obesity-induced insulin resistance. Proc Natl Acad Sci U S A 105:1739-1744

43. Handschin C, Chin S, Li P et al (2007) Skeletal muscle fibertype switching, exercise intolerance, and myopathy in PGC1alpha muscle-specific knock-out animals. J Biol Chem 282:30014-30021

44. Farese RV, Sajan MP, Yang H et al (2007) Muscle-specific knockout of PKC-lambda impairs glucose transport and induces metabolic and diabetic syndromes. J Clin Invest 117:2289-2301

45. Chin ER (2005) Role of $\mathrm{Ca}^{2+} /$ calmodulin-dependent kinases in skeletal muscle plasticity. J Appl Physiol 99:414-423

46. Hirosumi J, Tuncman G, Chang L et al (2002) A central role for JNK in obesity and insulin resistance. Nature 420:333-336

47. Kim JK, Kim YJ, Fillmore JJ et al (2001) Prevention of fatinduced insulin resistance by salicylate. J Clin Invest 108:437-446

48. Kramer HF, Goodyear LJ (2007) Exercise, MAPK, and NFkappaB signaling in skeletal muscle. J Appl Physiol 103:388-395

49. Hakimi P, Yang J, Casadesus G et al (2007) Overexpression of the cytosolic form of phosphoenolpyruvate carboxykinase (GTP) in skeletal muscle repatterns energy metabolism in the mouse. J Biol Chem 282:32844-32855

50. Lagouge M, Argmann C, Gerhart-Hines Z et al (2006) Resveratrol improves mitochondrial function and protects against metabolic disease by activating SIRT1 and PGC1alpha. Cell 127:1109-1122

51. Wang YX, Zhang CL, Yu RT et al (2004) Regulation of muscle fiber type and running endurance by PPARdelta. PLoS Biol 2: e294 
52. Coste A, Louet JF, Lagouge M et al (2008) The genetic ablation of SRC-3 protects against obesity and improves insulin sensitivity by reducing the acetylation of PGC- $1 \alpha$. Proc Natl Acad Sci U S A 105:17187-17192

53. Muoio DM, MacLean PS, Lang DB et al (2002) Fatty acid homeostasis and induction of lipid regulatory genes in skeletal muscles of peroxisome proliferator-activated receptor (PPAR) alpha knock-out mice. Evidence for compensatory regulation by PPAR delta. J Biol Chem 277:26089-26097

54. Wang YX, Lee CH, Tiep S et al (2003) Peroxisome-proliferatoractivated receptor delta activates fat metabolism to prevent obesity. Cell 113:159-170

55. Narkar VA, Downes M, Yu RT et al (2008) AMPK and PPARdelta agonists are exercise mimetics. Cell 134:405-415

56. Vanttinen M, Nuutila P, Kuulasmaa $T$ et al (2005) Single nucleotide polymorphisms in the peroxisome proliferatoractivated receptor delta gene are associated with skeletal muscle glucose uptake. Diabetes 54:3587-3591

57. Stefan N, Thamer C, Staiger H et al (2007) Genetic variations in PPARD and PPARGC1A determine mitochondrial function and change in aerobic physical fitness and insulin sensitivity during lifestyle intervention. J Clin Endocrinol Metab 92:1827-1833

58. Oliver WR Jr, Shenk JL, Snaith MR et al (2001) A selective peroxisome proliferator-activated receptor delta agonist promotes reverse cholesterol transport. Proc Natl Acad Sci U S A 98:5306-5311

59. Riserus U, Sprecher D, Johnson T et al (2008) Activation of peroxisome proliferator-activated receptor (PPAR) delta promotes reversal of multiple metabolic abnormalities, reduces oxidative stress, and increases fatty acid oxidation in moderately obese men. Diabetes 57:332-339

60. Fritz T, Kramer DK, Karlsson HK et al (2006) Low-intensity exercise increases skeletal muscle protein expression of PPARdelta and UCP3 in type 2 diabetic patients. Diabetes Metab Res Rev 22:492-498

61. Steinberg GR, Jorgensen SB (2007) The AMP-activated protein kinase: role in regulation of skeletal muscle metabolism and insulin sensitivity. Mini Rev Med Chem 7:519-526

62. Drew BG, Duffy SJ, Formosa MF et al (2009) High-density lipoprotein modulates glucose metabolism in patients with type 2 diabetes. Circulation 119:2103-2111

63. Kraus WE, Houmard JA, Duscha BD et al (2002) Effects of the amount and intensity of exercise on plasma lipoproteins. N Engl J Med 347:1483-1492

64. Buhl ES, Jessen N, Pold R et al (2002) Long-term AICAR administration reduces metabolic disturbances and lowers blood pressure in rats displaying features of the insulin resistance syndrome. Diabetes 51:2199-2206

65. Watt MJ, Steinberg GR, Chen ZP, Kemp BE, Febbraio MA (2006) Fatty acids stimulate AMP-activated protein kinase and enhance fatty acid oxidation in L6 myotubes. J Physiol 574:139-147

66. Garcia-Roves P, Huss JM, Han DH et al (2007) Raising plasma fatty acid concentration induces increased biogenesis of mitochondria in skeletal muscle. Proc Natl Acad Sci U S A 104:10709-10713

67. Drew BG, Kingwell BA (2008) Acadesine, an adenosineregulating agent with the potential for widespread indications. Expert Opin Pharmacother 9:2137-2144

68. Boon H, Bosselaar M, Praet SF et al (2008) Intravenous AICAR administration reduces hepatic glucose output and inhibits whole body lipolysis in type 2 diabetic patients. Diabetologia 51:18931900

69. Zhou G, Myers R, Li Y et al (2001) Role of AMP-activated protein kinase in mechanism of metformin action. J Clin Invest 108:1167-1174
70. Baur JA, Sinclair DA (2006) Therapeutic potential of resveratrol: the in vivo evidence. Nat Rev Drug Discov 5:493-506

71. Hou X, Xu S, Maitland-Toolan KA et al (2008) SIRT1 regulates hepatocyte lipid metabolism through activating AMP-activated protein kinase. J Biol Chem 283:20015-20026

72. Puigserver P, Wu Z, Park CW, Graves R, Wright M, Spiegelman BM (1998) A cold-inducible coactivator of nuclear receptors linked to adaptive thermogenesis. Cell 92:829-839

73. Jager S, Handschin C, St-Pierre J, Spiegelman BM (2007) AMPactivated protein kinase (AMPK) action in skeletal muscle via direct phosphorylation of PGC-1alpha. Proc Natl Acad Sci U S A 104:12017-12022

74. Lan F, Cacicedo JM, Ruderman N, Ido Y (2008) SIRT1 modulation of the acetylation status, cytosolic localization, and activity of LKB1. Possible role in AMP-activated protein kinase activation. J Biol Chem 283:27628-27635

75. Hawley SA, Boudeau J, Reid JL et al (2003) Complexes between the LKB1 tumor suppressor, STRAD alpha/beta and $\mathrm{MO} 25 \mathrm{alpha} / \mathrm{beta}$ are upstream kinases in the AMP-activated protein kinase cascade. J Biol 2:28

76. Feige JN, Lagouge M, Canto C et al (2008) Specific SIRT1 activation mimics low energy levels and protects against dietinduced metabolic disorders by enhancing fat oxidation. Cell Metab 8:347-358

77. Milne JC, Lambert PD, Schenk S et al (2007) Small molecule activators of SIRT1 as therapeutics for the treatment of type 2 diabetes. Nature 450:712-716

78. Pearson KJ, Baur JA, Lewis KN et al (2008) Resveratrol delays agerelated deterioration and mimics transcriptional aspects of dietary restriction without extending life span. Cell Metab 8:157-168

79. Civitarese AE, Carling S, Heilbronn LK et al (2007) Calorie restriction increases muscle mitochondrial biogenesis in healthy humans. PLoS Med 4:e76

80. Mathai AS, Bonen A, Benton CR, Robinson DL, Graham TE (2008) Rapid exercise-induced changes in PGC-1alpha mRNA and protein in human skeletal muscle. J Appl Physiol 105:10981105

81. Pilegaard H, Saltin B, Neufer PD (2003) Exercise induces transient transcriptional activation of the PGC-1alpha gene in human skeletal muscle. J Physiol 546:851-858

82. Fanelli M, Filippi E, Sentinelli F et al (2005) The Gly482Ser missense mutation of the peroxisome proliferator-activated receptor gamma coactivator-1 alpha (PGC-1 alpha) gene associates with reduced insulin sensitivity in normal and glucoseintolerant obese subjects. Dis Markers 21:175-180

83. Andrulionyte L, Peltola P, Chiasson JL, Laakso M (2006) Single nucleotide polymorphisms of PPARD in combination with the Gly482Ser substitution of PGC-1A and the Pro12Ala substitution of PPARG2 predict the conversion from impaired glucose tolerance to type 2 diabetes: the STOP-NIDDM trial. Diabetes 55:2148-2152

84. Handschin C, Spiegelman BM (2008) The role of exercise and PGC1alpha in inflammation and chronic disease. Nature 454:463-469

85. Graham TE, Battram DS, Dela F, El-Sohemy A, Thong FS (2008) Does caffeine alter muscle carbohydrate and fat metabolism during exercise? Appl Physiol Nutr Metab 33:1311-1318

86. Nayler WG, Dresel PE (1984) $\mathrm{Ca}^{2+}$ and the sarcoplasmic reticulum. J Mol Cell Cardiol 16:165-174

87. Raney MA, Turcotte LP (2008) Evidence for the involvement of CaMKII and AMPK in $\mathrm{Ca}^{2+}$-dependent signaling pathways regulating FA uptake and oxidation in contracting rodent muscle. J Appl Physiol 104:1366-1373

88. Wright DC, Geiger PC, Han DH, Jones TE, Holloszy JO (2007) Calcium induces increases in peroxisome proliferator-activated receptor gamma coactivator-1alpha and mitochondrial biogenesis 
by a pathway leading to p38 mitogen-activated protein kinase activation. J Biol Chem 282:18793-18799

89. Murase T, Haramizu S, Shimotoyodome A, Tokimitsu I, Hase T (2006) Green tea extract improves running endurance in mice by stimulating lipid utilization during exercise. Am J Physiol Regul Integr Comp Physiol 290:R1550-R1556

90. Collins QF, Liu HY, Pi J, Liu Z, Quon MJ, Cao W (2007) Epigallocatechin-3-gallate (EGCG), a green tea polyphenol, suppresses hepatic gluconeogenesis through 5'-AMP-activated protein kinase. J Biol Chem 282:30143-30149

91. Hwang JT, Ha J, Park IJ et al (2007) Apoptotic effect of EGCG in HT-29 colon cancer cells via AMPK signal pathway. Cancer Lett 247:115-121

92. Gomez-Cabrera MC, Domenech E, Romagnoli M et al (2008) Oral administration of vitamin $\mathrm{C}$ decreases muscle mitochondrial biogenesis and hampers training-induced adaptations in endurance performance. Am J Clin Nutr 87:142-149

93. Carey AL, Steinberg GR, Macaulay SL et al (2006) Interleukin-6 increases insulin-stimulated glucose disposal in humans and glucose uptake and fatty acid oxidation in vitro via AMPactivated protein kinase. Diabetes 55:2688-2697
94. Bolster DR, Crozier SJ, Kimball SR, Jefferson LS (2002) AMPactivated protein kinase suppresses protein synthesis in rat skeletal muscle through down-regulated mammalian target of rapamycin (mTOR) signaling. J Biol Chem 277:23977-23980

95. Meley D, Bauvy C, Houben-Weerts JH et al (2006) AMPactivated protein kinase and the regulation of autophagic proteolysis. J Biol Chem 281:34870-34879

96. Miura S, Tomitsuka E, Kamei Y et al (2006) Overexpression of peroxisome proliferator-activated receptor gamma co-activator1alpha leads to muscle atrophy with depletion of ATP. Am J Pathol 169:1129-1139

97. Kingwell BA, Jennings GL (1993) Effects of walking and other exercise programs upon blood pressure in normal subjects. Med J Aust 158:234-238

98. Church T, Blair SN (2009) When will we treat physical activity as a legitimate medical therapy...even though it does not come in a pill? Br J Sports Med 43:80-81

99. Hawley JA, Holloszy JO (2009) Exercise: It's the real thing! Nutr Rev 67:172-178

100. Richter EA, Kiens B, Wojtaszewski JF (2008) Can exercise mimetics substitute for exercise? Cell Metab 8:96-98 